ISSN: $2317-8957$

Volume 6, Number 1, Jun 2018

\title{
DESENVOLVIMENTO DE BIORREATORES DE BANCADA PARA BIORREMEDIAÇÃO DE SOLO CONTAMINADO COM DIESEL B5
}

\author{
FRANCISCO MACIEL MONTICELI* ${ }^{1}$, DENISE CELESTE GODOY DE ANDRADE RODRIGUES ${ }^{2}$, SERGIO \\ MACHADO CORRÊA ${ }^{2}$
}

${ }^{1}$ Faculdade de Engenharia Guaratinguetá, Universidade Estadual Paulista (Unesp), SP, Brasil. Departamento de Materiais e Tecnologia.

${ }^{2}$ Faculdade de Tecnologia, Universidade do Estado do Rio de Janeiro, RJ, Brasil.

* Corresponding author: Av. Dr. Ariberto Pereira da Cunha, No. 333, Guaratinguetá, São Paulo, 12516-410, Brasil. francisco monticelli@ hotmail.com, phone: +551231232865

\section{RESUMO}

Derivados de petróleo são uma das principais fontes de energia e, ao mesmo tempo, uma fonte de poluição de elevado impacto mundial. O uso crescente de automóveis fez com que ocorresse um proporcional aumento do número de postos de abastecimento. Tendo-se em vista a introdução da mistura diesel/biodiesel na matriz energética brasileira, faz necessário estudar a remediação deste contaminante no solo. Os biorreatores são sistemas fechados de tratamento e de biorremediação onde ocorre um maior controle sobre as variantes do sistema. Apresentam também, a possibilidade de coleta e tratamento de gases produzidos durante a degradação dos agentes contaminantes. O presente trabalho teve como objetivo projetar biorreatores de bancada e avaliar a capacidade de remediação de diferentes condições experimentais de solo contaminado com diesel B5. Os biorreatores foram construídos de garrafa de 1 litro de capacidade, provido de dreno inferior, entrada de ar lateral, camada de brita para espalhamento do ar de entrada, tela fina de nylon e vedação de silicone. A vazão de ar injetada no sistema foi controlada por rotâmetros, com injeção de ar durante intervalos de tempo contínuo. O solo foi artificialmente contaminado com 5\% de diesel B5 e a umidade controlada em $50 \%$ da capacidade de campo estimada (30,5\%). Os nutrientes foram adicionados a fim de se estabelecer relação nutricional C:N:P de 100:10:1 e o microcosmo recebeu o consórcio microbiano. Os tratamentos empregados foram atenuação natural monitorada (sem nutrientes); bioestímulo (com nutrientes) e bioestímulo com bioaumento (com nutrientes e consórcio) realizados em duplicata, com e sem aeração. Considerando os resultados encontrados, conclui-se que os biorreatores projetados podem ser empregados em estudos de biorremediação de solo. Foi demonstrado que a descontaminação do solo naturalmente apresentou baixo nível de remediação, comprovando a necessidade de metodologias de biorremediação de solo com maior eficiência. O sistema de aeração foi um fator primordial na contribuição da remediação do solo contaminado em todas as condições experimentais. Conclui-se que o sistema com ajuste nutricional, com bioaumento e com sistema de aeração dos biorreatores proporcionaram a biorremediação dos n-alcanos em mais de $80 \%$ durante 120 dias, sendo o melhor sistema de biorremediação.

Palavras-chave: Biorremediaçã. Biorreatores. Diesel B5. Solo contaminado.

\begin{abstract}
Petroleum derivatives are the main energy sources and a potentially pollution source. The increasing use of automobiles generated a proportional increase in gas stations number. With the introduction of the diesel / biodiesel mixture in the Brazilian energy matrix, resulted in increased bioremediation studies of this contaminated soil. Bioreactors are closed systems for variables and treatment control. In addition, it has the possibility to collect the gases produced during the contaminants degradation and treat it. This study aimed to design bioreactors bench and evaluate remediation ability of different experimental conditions of soil contaminated with diesel B5. The bioreactors were constructed using a bottle, provided with bottom drain, air inlet pipe, crushed stone layer to scattering inlet air, thin nylon screen and silicone for sealing. The air flow injected into the system was controlled by rotameters, with flow injection controlled during continuous time intervals. The soil was artificially contaminated with $5 \%$ B5 diesel and the humidity was controlled at $50 \%$ of the estimated field capacity $(30.5 \%)$. The nutrients were added to establish nutritional relation C: N: P for 100: 10: 1 and the microorganism received microbial consortium. The tested treatment were monitored Natural Attenuation (no nutrients); biostimulation (with nutrients); biostimulation with bioaugmentation (with nutrients and consortium) with and without air flow. Considering the results found, it was concluded that the projected bioreactors can be used in soil bioremediation studies. It has been demonstrated that naturally soil decontamination presented low level of remediation, proving the need for soil bioremediation methodologies with greater yield. Asystem was a primary factor to remediation contribution of the contaminated soil. It can be concluded that the system with nutritional adjustment, with bioaumento and with aeration system provided the bioremediation more than $80 \%$ during 120 days, being the best bioremediation system.
\end{abstract}

Key words: Bioremediation. Bioreactors. B5 Diesel. Contaminated soil.

\section{INTRODUÇÃO}

Devido ao aumento da população e crescimento tecnológico, evidenciados no final do último século, houve uma aumento na necessidade do consumo de energia e matéria prima que, consequentemente, gerou no aumento da poluição do meio ambiente. Todo ecossistema deveria estar balanceado e em equilíbrio. $\mathrm{O}$ aumento da quantidade de resíduos e poluentes resultaria na ocorrência do descontrole da equação natural, podendo gerar graves risco para os seres viventes e para o meio ambiente (Dantas et al., 2009; Toledo et al., 2011). $\mathrm{O}$ aumento da população gerou um 
aumento dos automóveis e viagens aeronáuticas comerciais que, por sua vez, gerou uma crescente proporcional do consumo de combustíveis. $\mathrm{O}$ resultado foi o aumento dos postos de abastecimento, principalmente para veículos automotores. O local de armazenamento mais comum nos postos são os chamados Sistemas de Armazenamento Subterrâneo de Combustível (SASC), ou seja, sistemas subterrâneos abaixo dos postos. Tais sistemas podem gerar graves acidentes de contaminação de solo e lençóis freáticos por meio de qualquer vazamentos (Simões, 2000; Bento et al., 2005; Mulligan, 2005; Cintra, 2013).

A CETESB apresentou a existência de mais de 2 mil incidentes com área contaminadas em na região sudeste, onde $77 \%$ destas áreas foram relacionadas com contaminação a partir de postos de combustíveis (CETESB, 2016). Torres et al. (2002) demonstrou os elevados teores de hidrocarbonetos aromáticos no monitoramento dos sedimentos do médio Paraíba do Sul, provenientes de vazamento de grande armazenamentos de combustíveis, entre outros.

A contaminação de solo pode ser realizada a partir de agentes gasosos, sólidos ou líquidos, mediante a possibilidade de impregnação das partículas por meio do soluto. Há estimativas de um aumento de cerca de 30 vezes a quantidade de poluição de certas áreas devido aos riscos de trabalho e crescimento populacional (Rinklebe et al., 2010; Souza et al., 2011; Deon et al., 2012).

O petróleo ou derivados de petróleo está entre os onze maiores agentes poluentes em todo o mundo, provenientes de acidentes durante sua extração, transporte, armazenagem e processamento. Os efeitos ecológicos são devastadores, pois é extremamente difícil controlar e realizar a remoção do petróleo depois de entornado, principalmente quando ocorre na água. Ainda tendo como grave consequência a geração de outros tipos de agentes poluentes como monóxido de carbono (exemplo: reação da combustão da gasolina), que também está na lista dos onze maiores poluentes (Nunes et al.,
2012; Aguiar et al., 2012; Cunha, 2014).

Devida à elevada toxidade dos derivados de petróleo, a contaminação do solo, em especial, pode gerar graves danos à saúde populacional, bacias hídricas, alteração de aspectos do solo, sem contar com a morte de fauna e flora (Finotti et al., 2001; Nunes et al., 2012; Aguiar et al., 2012; Cunha, 2014).

A biorremediação é uma prática do setor ambiental que tem sido cada vez mais importante e utilizada em estudos e experimentos visando o controle da interação ecológica natural e humana (Baheri et al., 2002; Diplock et al., 2009). A relevância do tema se dá pela qualidade de vida e qualidade ambiental através da biorremediação, visando o reparo de possíveis acidentes que poderiam prejudicar a saúde humana e o bem estar social, com o objetivo final de obter os mesmos padrões antes da contaminação (Baheri et al., 2002; Campos, 2014; Braga et al., 2014).

Existem diversos tipos de remediação de solo, que podem variar de acordo com fatores e condições do solo contaminado. $\mathrm{Na}$ qual é necessário a medição da concentração de contaminação, estruturas e componentes que constituem os agentes contaminadores. Por outro lado, análises como tempo de ação para remediação, eficiência e custos devem ser avaliadas para encontrar o tipo de remediação adequado para determinada situação e realidade. Técnicas têm sido estudadas e aprimoradas buscando o melhor desempenho a baixo custo. A Figura 1 mostra as principais técnicas de tratamento descritas na literatura (Baheri et al., 2002; Pereira et al., 2009; Diplock et al., 2009; Braga et al., 2014).

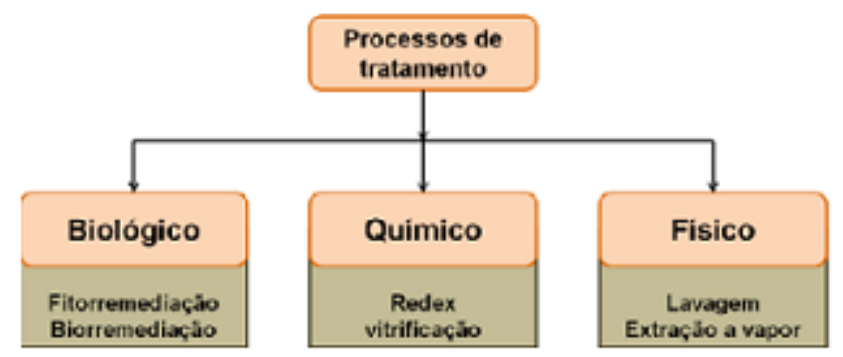

Figura 1. Tipos de tratamentos de solo. 
A utilização do processo de tratamento por biorremediação microbiana pode representar vantagens em eficiência (Bento et al., 2005; Mulligan, 2005; Pereira et al., 2012; Weber e Santos, 2013). Estudos mostram que tal tratamento pode ser realizado por bioestímulo, que trata de equalizar as relações nutricionais de $\mathrm{C}: \mathrm{N}: \mathrm{P}$, e bioaumento que se refere a adição de bio preparada para aumentar a população microbianas com o objetivo de diminuir os n-alcanos presente em derivado de petróleo, diminuindo assim porcentagem de contaminação (Xu et al., 2003; Jacques et al., 2007; Pereira et al., 2009; Diplock et al., 2009).

Os biorreatores são sistemas fechados de tratamento que podem realizar o tratamento de biorremediação, com maior controle sobre as variantes do sistema, tais como temperatura e umidade. Além disso, apresentam a possibilidade de coletar e tratar gases produzidos durante a degradação dos contaminantes. De acordo com o volume de solo contaminado a ser tratado, os biorreatores podem necessitar de um espaço reduzido em comparação com outros métodos de tratamento, facilitando o estudo em laboratórios de bancada (Jorgensen et al., 2000; Brasileiro et al., 2013).

O objetivo principal do presente trabalho foi encontrar o método mais adequado com melhores resultados para uma remediação de solo contaminado com diesel B5 utilizando biorreatores de baixo custo.

Para isso, foi desenvolvido e avaliado a viabilidade da utilização de biorreatores de bancada para pesquisa de biorremediação de solos contaminados com diesel B5. Em seguida, realizada uma análise comparativa da capacidade temediadora de microrganismos isolados de solo de postos de combustíveis na recuperação de solos contaminados por mistura diesel/biodiesel em diferentes condições de biorremediação. As divisões dos três tipos de biorreatores (atenuação natural, bioestímulo e bioestímulo com bioaumento) foram realizadas com o propósito da obtenção de uma análise comparativa de biorremediação de solo contaminado e qual a influência que o bioestímulo e o aumento causam nos resultados de remediação, avaliando o meio ótimo e com menor custo para obtenção dos resultados esperados. Resalinado, por fim, todo o experimento em duplicata (sistemas aerado e não aerado), com a finalidade de avaliar a influência da aeração na biorremediação.

\section{MATERIAIS E MÉTODOS}

O solo utilizado nos experimentos foi, inicialmente, um solo limpo fornecido pela EMBRAPA-SOLOS. Na Tabela 1 estão expostos as análises física e química do solo.

Tabela 1. Caracterização físico-química e microbiológica do solo estudado antes da contaminação.

\begin{tabular}{|c|c|c|}
\hline \multicolumn{2}{|c|}{ PARÂMETROS } & VALORES \\
\hline \multirow{3}{*}{ Granulometria $(\%)$} & Areia & 744 \\
\hline & Silte & 156 \\
\hline & Argila & 100 \\
\hline \multicolumn{2}{|c|}{ Capadidade de campo (\%) } & 61 \\
\hline \multicolumn{2}{|c|}{ Umidade (\%) } & 1,36 \\
\hline \multirow{6}{*}{$\begin{array}{l}\text { Complexo sortivo } \\
\left.\text { (comol. } \mathrm{Kg}^{-1}\right)\end{array}$} & $\mathrm{Ca}^{2+}$ & 1,2 \\
\hline & $\mathrm{Mg}^{2+}$ & 0.9 \\
\hline & $\mathrm{K}^{+}$ & 0,58 \\
\hline & $\mathrm{Na}^{+}$ & 0.01 \\
\hline & $\mathrm{Al}^{3+}$ & 0.1 \\
\hline & $\mathrm{H}+$ & 3,9 \\
\hline \multicolumn{2}{|c|}{$\begin{array}{ll}\mathrm{pH} & \text { Agua }\end{array}$} & 5,7 \\
\hline \multicolumn{2}{|c|}{$\begin{array}{l}\text { Carbon o orgânico total ( } \mathrm{g} \\
\left.\mathrm{Kg}^{1}\right)\end{array}$} & 11,4 \\
\hline \multicolumn{2}{|c|}{ Nitrogênio total $\left(\mathrm{g} \mathrm{Kg}^{\mathrm{I}}\right)$} & 1.7 \\
\hline \multicolumn{2}{|c|}{ Fósforo assimilávd (mg Kg $\left.\mathrm{m}^{-1}\right)$} & 28 \\
\hline \multicolumn{2}{|c|}{$\begin{array}{c}\text { População Heterotrófica total } \\
\text { (UFC g ge solo) }\end{array}$} & $9.51 \times 10^{6}$ \\
\hline
\end{tabular}

Este trabalhoutilizou como contaminante o diesel B5, que segundo site BIODISELBR (2014) é constituído por 5\% de biodiesel e 95\% de diesel. O solo foi contaminado com $5 \%$ de diesel B5 e deixado em repouso por uma semana em temperatura ambiente, a fim de eliminar as substâncias voláteis.

O parâmetro que pode ser definido como a quantidade máxima de água que um solo pode reter em condições normais, também conhecida como capacidade de campo, foi de 30,5\%. $50 \%$ da capacidade de campo é a utilização nos experimentos de biodegradação, os quais estariam próximo ao meio da faixa ótima citada na literatura (Pereira et al., 2002). 
A relação de nutrientes foi baseada no experimento de Bento et al. (2005), utilizando-se uma solução dos sais $\left(\mathrm{NH}_{4}\right)_{2} \mathrm{SO}_{4}$ e $\mathrm{K}_{2} \mathrm{HPO}_{4}$ como fontes de nitrogênio e fósforo, respectivamente. Com a introdução do óleo diesel B5, ou seja, com a entrada de uma nova fonte de carbono a proporção foi alterada, considerando que o teor de carbono orgânico proveniente do contaminante seria de $85 \%$ da massa total do contaminante adicionada (p. $\left.\mathrm{p}^{-1}\right)$, que foi de 50 g. $\mathrm{Kg}^{-1}$ de solo. Desse modo, para a execução dos processos de biorremediação propostos, houve a necessidade do acréscimo desses nutrientes no solo. A adição da solução nutriente foi realizada no início do experimento, obedecendo às condições de cada microcosmo.

Preparo do Inoculo: O consórcio microbiano (inoculo) foi preparado adicionandose $20 \mathrm{~g}$ do solo, proveniente de um posto de combustível da cidade de Resende - RJ, a frasco erlenmeyer $(250 \mathrm{~mL}$ ) contendo $2 \%$ (massa por volume) de biodisel e $100 \mathrm{~mL}$ de meio BushnellHass (BH) - 0,20 g.L. ${ }^{-1} \mathrm{MgSO}_{4} \cdot{ }_{7} \mathrm{H} 2 \mathrm{O}, 0,02$ g.L. ${ }^{1} \mathrm{CaCl}_{2} .2 \mathrm{H}_{2} \mathrm{O}, 1,00$ g.L ${ }^{-1} \mathrm{KH}_{2} \mathrm{PO}_{4}, 1,00$ g.L ${ }^{-1}$ $\mathrm{K}_{2} \mathrm{HPO}_{4}, 1,00$ g.L $\mathrm{L}^{-1} \mathrm{NH}_{4} \mathrm{NO}_{3}, 0,05$ g.L ${ }^{-1} \mathrm{FeCl}_{3} \mathrm{e}$ $\mathrm{H}_{2} \mathrm{O}$ - e mantido sob agitação durante $48 \mathrm{~h}$.

Foi possível conhecer todas as características do solo através da caracterização do solo, que constituiram em: análises físicoquímicas; capacidade de campo, umidade e análise de micro-organismos heterotróficos totais, realizadas no Laboratório de Microbiologia - FAT/UERJ.

O esquema utilizado para estudo, apresentado na Figura 2, foi com a seguinte divisão: ATN (atenuação natural) somente com solo contaminado; BE (bioestímulo) com solo contaminado e correção nutricional $\mathrm{C}: \mathrm{N}: \mathrm{P}$ de 100:10:1 (Molina-Barahona et al., 2004), para a correção da relação nutricional foi utilizado sulfato de amônia $\left(\mathrm{NH}_{4}\right)_{2} \mathrm{SO}_{4}$ e Fosfato de Potássio $\mathrm{K}_{2} \mathrm{HPO}_{4}$; BEA (bioestímulo com bioaumento) com solo contaminado, correção nutricional da relação $\mathrm{C}: \mathrm{N}: \mathrm{P}$ e reinoculação de micro-organismos indígenas após enriquecimento e aclimatação em meio $\mathrm{BH}$ com $1 \%$ de diesel B5. A pesquisa foi realizada em duplicata com todos os dados mencionados na Tabela 2, onde foi possível realização de estudo com sistema aerado (ATN-AR, BE-AR, BEAAR) e sistemas sem aeração (ATN, BE, BEA).

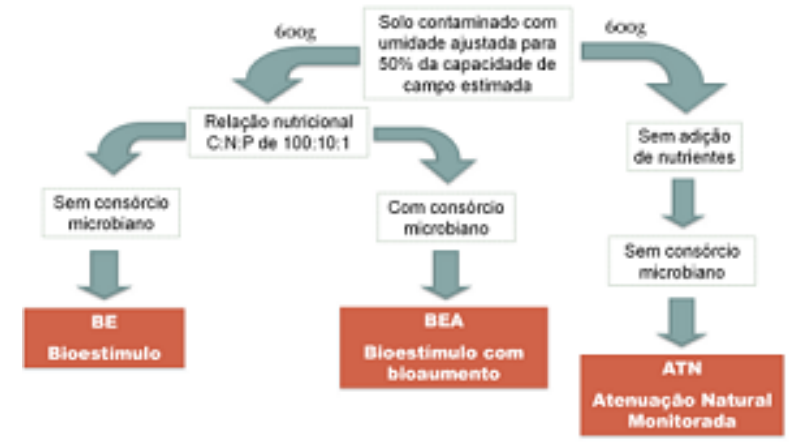

Figura 2. Esquema de trabalho dos biorreatores.

Tabela 2. Condições experimentais

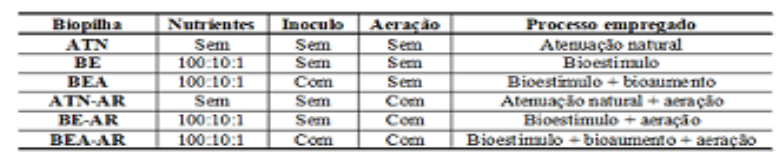

O solo foi controladamente contaminado em laboratório e separado em seis biorreatores. Os Biorreatores foram confeccionados com três suportes de garrafa de isopor com capacidade de 1 litro. De acordo com Figura 3, a saída inferior (espessura de aproximadamente $8 \mathrm{~mm}$ ), para coleta de possíveis lixiviados. Foram feitos alguns pequenos furos na parte superior para a saída de ar e, finalmente, um furo na lateral inferior, a cerca de $1 \mathrm{~cm}$ da base das garrafas por onde foi acoplada a tubulação de entrada de ar. O fluido ao escoar de baixo pra cima desloca o flutuador para cima, até uma posição onde ocorrerá o equilíbrio entre o peso do flutuador e as forças de arraste e de empuxo do fluido sobre o flutuador. Projetou-se uma camada de brita de aproximadamente 2 centímetros de altura para espalhamento do ar de entrada para os sistemas aerados, com o objetivo de evitar um caminho preferencial para o ar e para drenagem de possíveis lixiviados do sistema, separada da camada de solo a ser tratado por uma tela fina de nylon, onde a garrafa foi cortada e colocada esta 
tela pouco a cima da entrada de ar. A vedação do sistema foi feita com silicone.

Os biorreatores não aerados foram montados da mesma forma, porém sem o sistema e a entrada de ar, ilustrados na Figura 4. Um sistema de rotâmetros da marca Dwyer controlaram a vazão ( 0 à 1 L.min ${ }^{-1}$ ) e a entrada de ar iguais para os três sistemas. A bomba de ar conectada a um temporizador da marca ELCON modelo TE-30, realizando aeração 8 vezes por dia com duração de uma hora e intervalos de duas horas. A Figura 5 mostra todo o sistema de reatores montados em bancada.

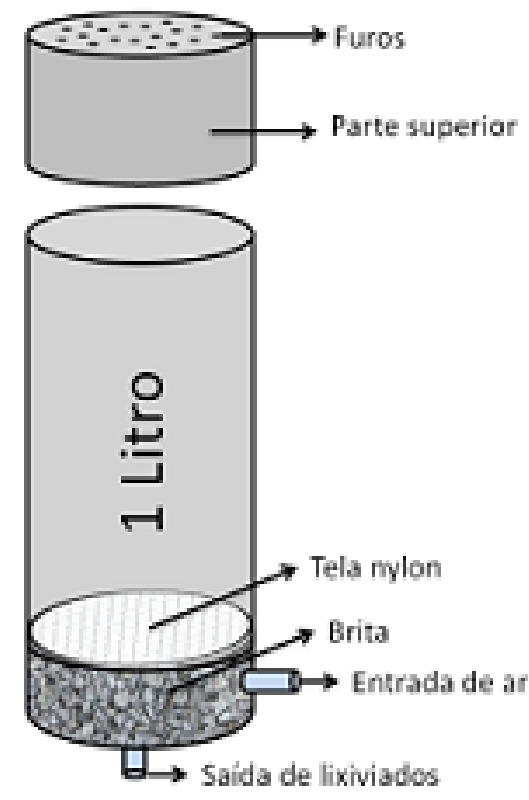

Figura 3. Esquema do biorreator.

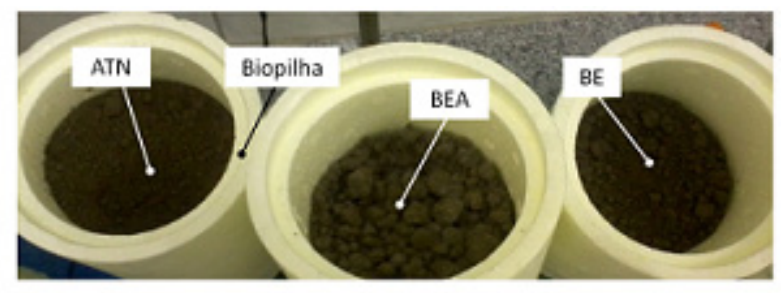

Figura 4. Vista superior dos biorreatores não aerados.

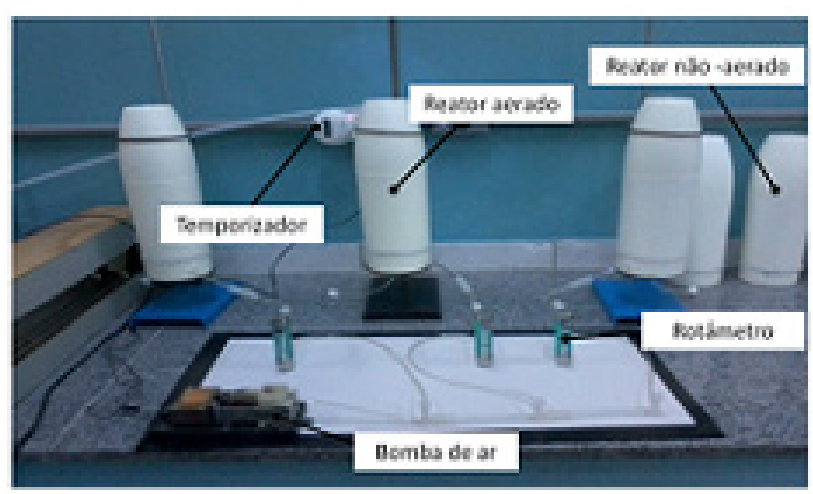

Figura 5. Sistema de Biorreatores aerados.

Mais detalhes do biorreator após a confecção (Figura 6), antes da utilização do solo para experimento, mostrando a entrada de ar inferior, a saída inferior de lixiviados e a vedação do biorreator com silicone (Figura 6A). $\mathrm{Na}$ figura a direita (Figura 6B) está exposta a tela de nylon que faz a separação entre o cascalho e a terra.

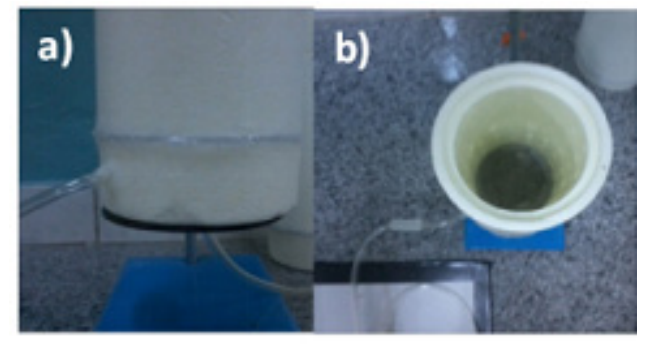

Figura 6. Ilustração do inferior do biorreator com a entrada de ar: a) saída de lixiviado e a vedação com silicone e b) interior do biorreator com a tela de separação de nylon.

Os biorreatores foram aerados pelo sistema, enquanto três deles ficaram estáticos para obtenção qualitativa das melhores especificações do sistema. Foi observado durante 120 dias, tendo sua umidade regulada uma vez por semana com água destilada estéril. Com relação ao $\mathrm{pH}$, o solo em estudo mostrouse neutro, não necessitando de correção, uma vez que a faixa ótima de $\mathrm{pH}$ citada na literatura para tal fim situa-se entre 6,5 a 8,5 (Jacques et al., 2010).

A técnica de Unidades Formadoras de Colônias (UFC) plaqueadas pour plate em meio PCA esterilizado em autoclave a 121 ${ }^{\circ} \mathrm{C}$ por 15 minutos (Colla et al., 2008). Os 
hidrocarbonetos Totais de Petróleo (HTP) foram extraídos do solo em extrator Soxhlet, apresentado na Figura 7, de acordo com a metodologia U.S.EPA 3540C (Ris, 2007). Os hidrocarbonetos extraíveis de petróleo foram analisados pela cromatografia Varian CP3800 com detector de ionização de chama (CG-DIC). Os compostos alvos para análise de HTP foram, principalmente, os n-alcanos na faixa de $\mathrm{C}_{10}$ a $\mathrm{C}_{36}$.

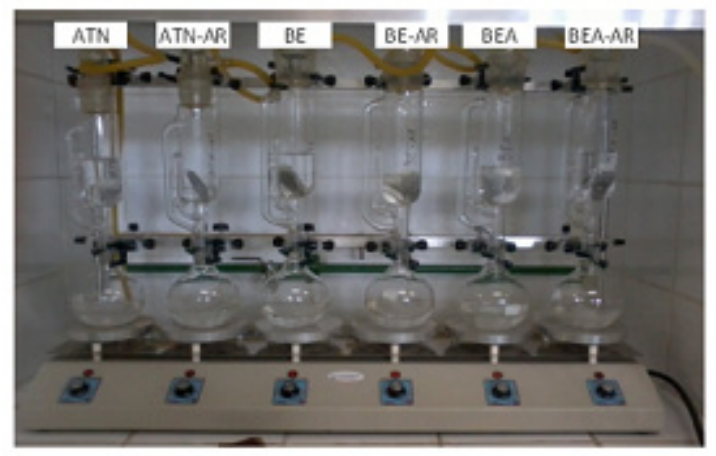

Figura 7. Extrator Soxhlet para amostra de cada reator.

\section{RESULTADOS}

A cada 30 dias as amostras foram analisadas em relação a presença dos microorganismos heterotróficos totais pela técnica UFC plaqueadas pour plate em meio PCA esterilizado em autoclave. Os resultados relativos ao crescimento dos micro-organismos heterotróficos totais e presença de n-alcanos encontram-se nas Tabela 3 e 4.

Tabela 3. Estimativa média dos micro-organismos heterotróficos totais (UFC.g -1 solo) nos intervalos 0, 30, 60, 90 e 120 dias de monitoramento do experimento.

\begin{tabular}{|c|c|c|c|c|c|}
\hline LAeatificaşäo & edias & 30 dias & 60 dias & 90 dias & 120 dias \\
\hline $\operatorname{ATN}$ & $9,85 \times 10^{5}$ & $1,84 \times 10^{4}$ & $8,75 \times 10^{\circ}$ & $1.92 \times 10^{7}$ & $1,92 \times 10^{\circ}$ \\
\hline $\mathrm{BE}$ & $9,75 \times 10^{9}$ & $1,83 \times 10^{\circ}$ & $5,15 \times 10^{\prime}$ & $1.87 \times 10^{7}$ & $1.85 \times 10^{7}$ \\
\hline BEA & $10,73 \times 10^{5}$ & $1,95 \times 10^{\circ}$ & $3,82 \times 10^{\circ}$ & $1,15 \times 10^{8}$ & $1,76 \times 10^{\circ}$ \\
\hline ATNAR & $5,60 \times 10^{5}$ & $1,06 \times 10$ & $1,11 \times 10^{4}$ & $9.64 \times 10^{7}$ & $2,19 \times 10^{\prime}$ \\
\hline$B E-A R$ & $10,02 \times 10^{5}$ & $7,72 \times 10^{0}$ & $5,32 \times 10^{\prime}$ & $1,20 \times 10^{7}$ & $1,53 \times 10^{\circ}$ \\
\hline BEAAR & $11,10 \times 10^{4}$ & $2,10 \times 10^{\circ}$ & $5.40 \times 10^{\circ}$ & $1.75 \times 10^{7}$ & $1.76 \times 10^{7}$ \\
\hline
\end{tabular}

Tabela 4. n-Alcanos presente no solo contaminado nos intervalos de 30, 60, 90 e 120 dias de experimento.

\begin{tabular}{ccccc}
\hline Identifkaça & 30 dias & 60 dias & 90 dias & 120 dias \\
\hline ATN & 6754 & 5497 & 5276 & 5341 \\
BE & 1624 & 1474 & 1031 & 645 \\
BEA & 2707 & 1412 & 2286 & 1293 \\
ATN.AR & 9251 & 5861 & 4433 & 3049 \\
BE-AR & 1563 & 1040 & 497 & 371 \\
BEA-AR & 1817 & 838 & 637 & 322 \\
\hline
\end{tabular}

Este experimento prevê o aumento de micro-organismos presentes no solo, visto que o maio está disponibilizando um ambiente controlado favorável para o seu crescimento. Cabe ressaltar que os micro-organismos heterotróficos são aqueles que utilizam compostos orgânicos como sua principal fonte de carbono, sendo os componentes de destaque na biodegradação de contaminantes orgânicos, gerando uma diminuição dos n-alcanos presentes no solo devido a contaminação. No solo, a maioria desses micro-organismos é representada por actinomicetos, fungos e bactérias (Moreira \& Siqueira, 2006).

O objetivo da divisão dos tipos de biorreatores foi de realizar uma análise do método mais eficiente, sendo possível uma melhor visualização do comportamento do crescimento e estabilização dos micro-organismos durante todos os dias de experimento nos gráficos apresentados nas Figuras 8 e 9.

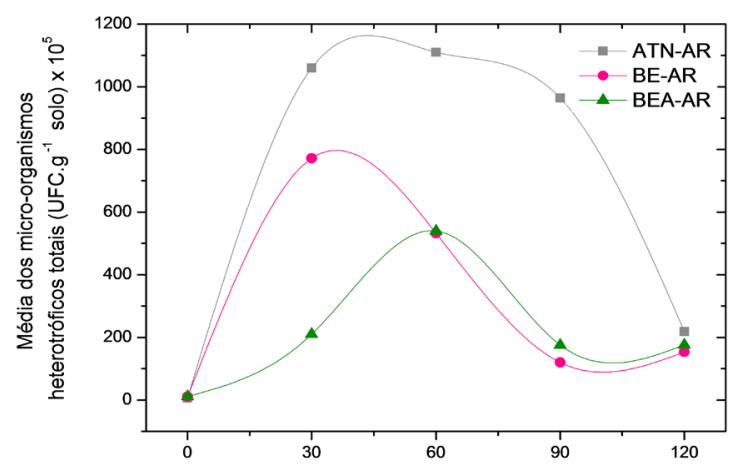

Figura 8. Comportamento do crescimento de microorganismos heterotróficos - aerado. 


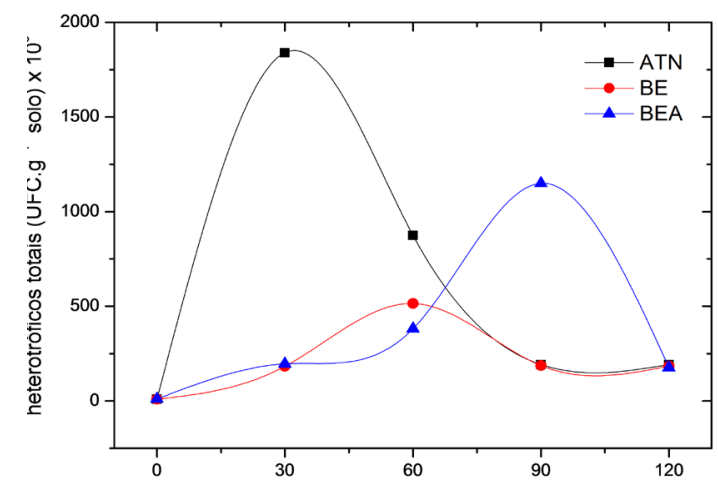

Figura 9. Comportamento do crescimento de microorganismos heterotróficos - não aerado.

Ocorre uma grande flutuação no crescimento de micro-organismos, principalmente devido as condições experimentais de nutrição do solo, adição microbiana e aeração no solo. Um fator que influenciou no crescimento dos microorganismos foi o tamanho do recipiente, fator pode ter limitado o crescimento dos mesmos gerando resultados próximos entre si no final dos 120 dias de experimentos.

Pode-se observar que houve crescimento dos micro-organismos durante os primeiros 60 dias de análise, sendo a quantidade máxima obtida da ordem de $10^{8} \mathrm{UFC}^{-g^{-1}}$ solo para as condições ATN e ANT-AR e 107 UFC.g $^{-1}$ solo para o restante das condições.

A explicação para a diminuição da quantidade de micro-organismos, evidenciada próximo ao dia 90 e estabilização baixa até o dia 120 , se dá ao fato de que a quantidade de massa nos reatores limitou o crescimento dos microorganismos. Prova dessa afirmativa pode se dar ao fato de que nos primeiros 30 dias houve um grande crescimento microbiano, principalmente nos sistemas aerados, após esse crescimento houve a estabilização microbiana e o equilíbrio de acordo com a diminuição dos n-alcanos.

Os resultados de n-alcanos encontrados nos sistemas não aerados foram semelhantes aos do sistema aerado, porém com menor percentual de diminuição de n-alcanos, devido ao menor crescimento dos micro-organismos. Já os sistemas aerados se mostraram bastante eficientes com uma diminuição de n-alcanos bastante linear, apresentado na Figura 10.

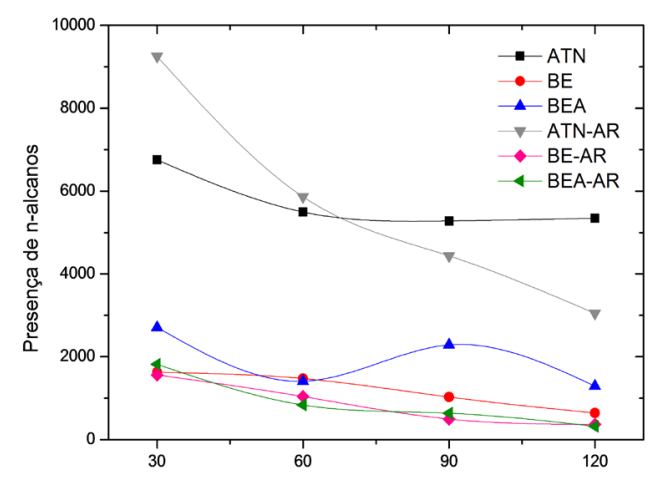

Figura 10. Presença de n-alcanos por tempo de análise.

Após um total de 120 dias de experimentos de bancada, apesar de concluir que o tamanho do biorreator pode ter limitado o crescimento dos micro-organismos e até mesmo impedido uma cotação de melhores resultados ou menor tempo de remediação, a degradação dos n-alcanos para cada biorreator revelaram resultados positivos. Os resultados de degradação para cada experimento mostram que a biorremediação foi bem sucedida.

O experimento sem nenhum tipo de adição microbiana e sem nenhuma melhoria nutricional, biorreator ATN e ATN-AR, resultaram em uma quantidade de n-alcanos bem maior do que o restante, pois sem o ajuste $\mathrm{C}: \mathrm{N}: \mathrm{P}$ houve maior porcentagem inicial de carbono presente no sistema. Mesmo com esse agravante apresentaram bons resultados, pois o aumento de micro-organismos foi considerável, embora os resultados obtidos através da degradação de n-alcanos foram somente de $21 \%$ do sistema não aerado. Já no sistema aerado foi de $67 \%$ de remediação. Em outras palavras, este fato prova que naturalmente ocorre uma descontaminação de $21 \%$ do solo e há um ganho considerável com a aeração do solo, aumentando para $67 \%$ de descontaminação. Isso implica que somente a aeração do sistema pode aumentar a eficiência de remediação em aproximadamente $45 \%$.

O biorreator com melhoria de relação nutricional, o bioestímulo BE e BE-AR obtiveram a mesma grandeza de crescimento 
AS\&T Volume 6, Number 1, Jun 2018

de micro-organismos, e ainda apresentaram resultados consideráveis de remediação, chegando a $60 \%$ e $76 \%$ de diminuição de n-alcanos, respectivamente.

A realização de bioestímulo e bioaumento, BEA e BEA-AR, apresentaram valores bem próximos aos reatores somente com bioestímulo tanto no aumento de microorganismos quanto na diminuição de n-alcanos, que foram de $52 \%$ e $82 \%$.

A diferença entre os tratamentos ATN, $\mathrm{BE}$ e BEA (sistemas não-aerados) mostrou que somente o ajuste nutricional dos biorreatores e proporcionou a biorremediação dos n-alcanos com resultado de $60 \%$ de remediação em um tempo de 120 dias.

$\mathrm{O}$ resultado do biorreator natural sem aeração apresentou baixa remediação, indicando que naturalmente a remediação tem um rendimento baixo e pode levar muito tempo para voltar ao seu estado natural.

Porém todos os sistemas aerados mostraram uma melhoria de remediação efetiva comparados aos sistemas não aerados. Com isso, é possível provar que a aeração é um importante fator no processo de biorremediação do solo contaminado com diesel B5. A aeração do sistema evidenciou que a remediação utilizando bioestímulo e bioaumento com a aeração (BEA-AR) é o sistema mais adequado, gerando o melhor resultado de biorremediação.

Considerando as tendências de diminuições de n-alcanos, evidenciado no gráfico da Figura 10, pode-se esperar maiores valores de remediação com o passar do tempo. Entretanto, levando em conta que houve uma diminuição dos micro-organismos devido a limitação de massa de solo, maiores valores de remediação do solo nas mesmas condições experimentais poderiam demorar um tempo muito maior.

Visando buscar melhores resultados, para estudos futuros, é proposta a realização de experimentos com sistemas de biorreatores maiores e com maior tempo de remediação, com o objetivo de encontrar resultados de biorremediação mais próximo aos $100 \%$.

\section{CONCLUSÕES}

Conclui-se que os biorreatores projetados podem ser empregados em estudos de biorremediação de solo para estudos em escala experimentais de laboratório, ainda com o fato da utilização de biorreatores serem eficientes e de baixo custo para experimento.

Considerando que os resultados com maior eficiência de remediação foram apresentados pelos sistemas com aeração, onde a utilização do mesmo gerou um aumento médio de $30 \%$ de remediação comparados aos sistemas não-aerados, conclui-se que a aeração do solo proporciona melhores condições de remediação do solo em todas as condições.

Com a utilização do sistema de aeração, todas as condições resultaram em remediação superior a $65 \%$, considerando que corre uma maior eficiência com reatores com biestímulo e eficiência ainda maior para adição de bio, resultando no crescimento de microorganismos e degradação de n-alcanos durante os 120 dias de análise. Como conclusão final, a disponibilização de um sistema com aeração, a metodologia mais adequada foi a utilização de biorreatores com bioaumento e bioestímulo, resultando em mais de $80 \%$ de rendimento em remediação do solo para seu estado natural, com sistema de biorreatores de baixo custo.

\section{AGRADECIMENTOS}

Ao Conselho Nacional de Desenvolvimento Científico e Tecnológico $(\mathrm{CNPq})$, sob o processo $\mathrm{n}^{\mathrm{o}} 113472 / 2010-$ 2 , pelo apoio e financiamento deste projeto.

\section{REFERÊNCIAS}

AGUIAR, C.R.C.; Barbosa, M.V.D.; Balieiro, F.C.; Gomes, M.M. 2012. Fitorremediação de solos contaminados por petróleo. Revista Trópica: Ciências Agrárias eBiológicas 6(1):3-9.

BAHERI, H.; MEYSAMI, P. 2002. Feasibility of fungi bioaugmentation in composting a flare pit soil. Journal of 
hazardous materials, B89: $279-286$.

BENTO, F.M.; CAMARGO, F.A.O; OKEKE, B.C.; FRANKENBERGER, W.T. 2005. Comparative bioremediation of soils contaminated with diesel oil by natural attenuation, biostimulation and bioaugmentation. Bioresource Technology 96: 1049-1055.

BIODIESEL BR. $2015<$ http://www. biodieselbr.com/biodiesel/definicao/o-que-ebiodiesel.htm> 13 Abr 2017.

BRAGA, T.G. et al. 2014. Biorremediação de solo contaminado com biodiesel de processo (biodiesel e glicerina): avaliação de condições operacionais empregando reatores rotativo e de bandeja. Blucher Chemical Engineering Proceedings 1(1): 34-37.

BRASILEIRO, P.P.F.; Souza Filho, G.L.; Silveira, G.N.M.; Rocha e Silva, N.M.P.; Luna, J.M.; Rufino, R.D.; Santos, W.A.; Sarubbo, L.A. 2013. Estudo da estabilidade do biossurfactante produzido em biorreator para biorremediação. In: I Congresso Internacional de Ciências Biológicas, II Congresso Nacional de Ciências Biológicas e VI Simpósio de Ciências Biológicas 1: 1-13.

CAMPOS, I.Z.A. 2014. A importância de tratamento de águas residuais através da biorremediação: uma análise principiológica. Revista de Filosofia do Direito, do Estado e da Sociedade 5(2): 46-58.

CETESB SOLO. 2016. Disponível em: http://www.cetesb.sp.gov.br/Solos/areas contaminadas/relação_areas.asp Acesso em Setembro 2016.

CINTRA, M. 2013. A crise do trânsito em São Paulo e seus custos. GV-executivo 12(2): 58-61.

COLLA, L.M.; PRIMAZ, A.L.; LIMA, M.; BERTOLIN, T.E.; COSTA, J.A.V.
2008. Isolamento e seleção de fungos para biorremediação a partir de solo contaminado com herbicidas triazínicos. Ciência Agrotecnologia Lavras 32(3): 809-813.

CUNHA, B.B. 2002. Degradação e conservação do meio ambiente, introdução a poluição. Módulo 2, 1: 45-47.

DE SOUZA, J.A.A.; RAMOS, M.M.; SOARES, A.A. 2011. Contaminação microbiológica do perfil do solo com esgoto sanitáriodoi: 10.4025/actascitechnol. v33i1. 5350. Acta Scientiarum Technology 33(1): 5-8.

DE TOLEDOI, G.I.F.M.; NARDOCCII, A.C. 2011. Poluição veicular e saúde da população: uma revisão sobre o município de São Paulo (SP), Brasil. Rev Bras Epidemiol 14(3): 445-54.

DEON, M.C. et al. 2012. Biorremediação de solos contaminados com resíduos oleosos através de bioaumentação e atenuação natural. Semina: Ciências Exatas e Tecnológicas 33(1): 73-82.

DANTAS, A.A.; NETO, E.L.B.; MELO, J.F.S.; NASCIMENTO, L.A. 2009. Avaliação da Percolação do Óleo Diesel, Água e Solução de Tensoativo em Solo Arenoso. $5^{\circ}$ Congresso Brasileiro de Pesquisa e Desenvolvimento em Petróleo e Gás 1:1-5.

FINOTTI, A.R.; CAICEDO, N.O.L. 2001. Contaminações subterrâneas com combustíveis derivados de petróleo: toxicidade e legislação brasileira. Revista Brasileira de Recursos Hídri$\cos 6(2): 29-46$.

DIPLOCK, E.E. 2009. Predicting bioremediation of hydrocarbons: laboratory to field scale. Environmental Pollution 157(6): 1831-1840.

JACQUES; R.J.S.; BENTO, F.M.; ANTONIOLLI, Z.I.; CAMARGO, F.A.O. 2007. Biorremediação de solos contaminados com hidrocarbonetos aromáticos policíclicos. Ciência Rural 37(4): 1192-1201. 
AS\&T Volume 6, Number 1, Jun 2018

MOREIRA, F.M.S.; SIQUEIRA, J.O. 2006. Mi-

Brasileira de Energia 9: 1-9. crobiologia e bioquímica do solo. 2 ed. Lavras: UFLA, $729 \mathrm{p}$.

MULLIGAN, C.N. 2005. Environmental applications for biosurfacts. Environmental Pollution 133: 183-198.

NUNES, G.B.; BARBOSA, A.F.F. 2012. Gestão dos resíduos sólidos provenientes dos derivados de petróleo em oficinas mecânicas da cidade de Natal/RN. Encontro Nacional de Educação, Ciência e Tecnologia/UEPB, 1: 1-11.

PEREIRA, C.A.; MARQUES, M.R.; PÉREZ, D.V. 2009. Avaliação da potencialidade de processos pseudofenton para remediação de solos contaminados por diesel. Química Nova 32(8): 2200-2202.

PEREIRA, L.T.C.; ARAÚJO, F.M.S.; SANTOS, L.C.; RAIMUNDO, R.S.; LEMOS, J.L.S. 2002. Biorremediação de solos impactados por óleo cru utilizando fungos filamentosos. Ln: Comunicação Técnica ao II Congresso Brasileiro de Iniciação Científica, S. Carlos - SP 1: 28-29.

PEREIRA, A.R.B.; DE FREITAS, D.A.F. 2012. Uso de micro-organismos para a biorremediação de ambientes impactados. Electronic Journal of Management, Education and Environmental Technology (REGET) 6(6): 995-1006.

RINKLEBE, J.; DURING, J.; OVERESCH, M.; LAING, G. J.; WENRICH, R.; STÄRK, H.; MOTHES, S. 2010. Dynamics of mercury fluxes and their controlling factors in large $\mathrm{Hg}$ polluted floodplain areas. Environmental Pollution 158(1): $308-318$.

RIS, C. 2007. U.S. EPA Health Assessment for Diesel Engine Exhaust: A Review. U.S. Environmental Protection Agency, Washington, 19(1): 229-239.

SIMÕES A.F. 2000. Emissões de CO 2 devido ao transporte aéreo no Brasil. SBPE, Revista

TORRES, J.P.M.; MALM, O.; VIEIRA, E.D.R.; JAPENGA, J.; KOOPMANS, G.F. 2002. Organic micropollutants on river sediments from Rio de Janeiro State, southeast Brazil, Cad. Saúde Pública 18(2): 477-488.

WEBER, B.D.; SANTOS, A.A. 2013. Utilização da Biorremediação como Ferramenta para o controle da Degradação Ambiental Causada pelo Petróleo e seus Derivados. Engenharia Ambiental: Pesquisa e Tecnologia 10(1): 1-13.

XU, R.; OBBARD. J.P. 2003 Effect of nutrient amendments on indigenous hydrocarbon biodegradation in oil-contaminated beach sediments, Journal Environment Qual 32: 1234-12 